\title{
Effect of Intradialytic Aerobic Exercise on C-Reactive Protein in End Stage Renal Disease Patients
}

\author{
Juwita Arum Mayangsari ${ }^{1}$, Imam Subadi ${ }^{2}$, Nuniek Nugraheni ${ }^{3}$, \\ Nunuk Mardiana ${ }^{4}$, Mochamad Thaha ${ }^{5}$ \\ ${ }^{1}$ Resident of Department of Physical Medicine and Rehabilitation \\ ${ }^{2,3}$ Physiatrist of Department of Physical Medicine and Rehabilitation \\ ${ }^{4,5}$ Internist of Department of Internal Medicine, \\ Dr. Soetomo Hospital/ Faculty of Medicine, University of Airlangga, Surabaya, Indonesia \\ corresponding author: juwita.arum.mayangsari-2017@fk.unair.ac.id
}

\begin{abstract}
Background: Patients with end-stage renal disease (ESRD) have a high baseline level of systemic inflammation. Serum C-Reactive Protein is an inflammatory marker that is widely used because it is more affordable and available in most hospitals in developing countries. Patients with ESRD tend to be less involved in physical activity when compared to sedentary patients in the same age group. The effect of intradialytic aerobic exercise on CRP levels has been widely studied, but the result studies about intradialytic aerobic exercise on CRP are still controversial.
\end{abstract}

Objectives: To evaluate the effect of a 12-week intradialytic aerobic exercise program on serum CRP levels in ESRD at the Hemodialysis Installation of Dr. Soetomo Hospital, Surabaya

Methods: The study was conducted from March to June 2021. Twenty-six ESRD patients divided into treatment and control groups were able to follow the study until the end. The treatment group did a low-intensity intradialytic exercise program for 12 weeks. Both groups were given education about inflammation in ESRD patients and continued drug therapy and diet as before. Serum CRP was checked before and after intervention.

Results: There was a decrease in the mean CRP after the intervention, from $0.33 \pm 0.33$ to $0.27 \pm 0.16 \mathrm{mg} / \mathrm{dL}$ but was no significant difference statistically $(\mathrm{p}=0.543)$ and the control group $(\mathrm{p}=0.08)$ and between the two groups $(\mathrm{p}=0.960)$.

Conclusion: Intradialytic aerobic exercise has no effect on serum levels of C-reactive protein in end-stage renal disease patients

Keywords: Intradialytic exercise; rehabilitation; ESRD; CKD; CRP; Inflammation

\section{Background}

The incidence of ESRD are estimated to exceed 2.5 million people and are projected to double to 5.4 million by 2030 (Bikbov et al., 2020). The Indonesian Nephrology Association (PERNEFRI) in the 11th Report of the Indonesian Renal Registry (IRR) reported an increase in the prevalence of ESRD in Indonesia by more than 50\% from 77,892 in 2017 to 132,142 in 2018 (PERNERFI, 2018). Patients with end-stage renal disease (ESRD) have a high baseline level of systemic inflammation. Systemic inflammation has implications for a number of morbidities including cardiovascular disease which is the most common cause of death in this 
population (Dungey et al., 2013). This group showed great improvement with exercise therapy. Exercise in ESRD patients has a positive effect on the effectiveness of dialysis, decreases C-reactive protein (CRP), decreases blood pressure and arterial stiffness and improves health-related quality of life (Heiwe and Jacobson, 2014).

ESRD patients tend to be less involved in physical activity when compared to sedentary patients in the same age group (Johansen et al., 2007). This group of patients is advised to do aerobic physical activity 5 times a week for at least 30 minutes (Levin et al., 2012). Intradialytic exercise is said to be the most ideal choice for ESRD patients because in addition to not requiring additional time, the patient is under the supervision of a doctor and a monitoring machine when performing intradialytic exercises and the possibility that intradialytic exercise can increase solute removal. Aerobic exercise with a foot cycle ergometer can be performed when the patient is undergoing haemodialysis without disturbing the arteriovenous fistula (AVF) in the hand connected to the HD device (Sheng et al., 2014).

The effect of aerobic exercise on CRP levels has been widely studied, but studies on intradialytic aerobic exercise on CRP are still controversial. Abdellah et al. (2020) and Groussard et al. (2015) stated that there was a decrease in serum CRP of ESRD patients after intra-dialysis exercise, while Suhardjono et al. (2019) did not find changes in serum hsCRP levels of ESRD patients after intradialytic exercise. This study aims to determine whether intradialytic aerobic exercise affects the CRP level of ESRD population.

\section{Materials and Method}

This study is a quasi-experimental pre-post randomized controlled group study. It was carried out from March 2021 to June 2021 and has obtained ethical eligibility from the Ethics Committee of Dr. Soetomo Hospital, Surabaya, with ethics number 0153/KEPK/II/202I. All activities ranging from recruitment, blood sampling and treatment during the study were carried out at the hemodialysis installation of Dr. Soetomo Hospital, Surabaya, Indonesia.

The sample size was calculated by hypothesis testing using a formula for difference in means. The sample size was determined based on the Lwanga and Lemeshow (1991) formula two-sided test for two population means. The minimum sample size in each group was 8 . In this study subjects were selected consecutively. The inclusion criteria were age 30-50 years, undergoing routine haemodialysis for at least 3 months, agree to participate in this study voluntarily by signing the consent form. Exclusion criteria included undergoing a routine aerobic exercise program 2 times a week, unstable heart disease, new stroke, resting systolic blood pressure less than 110 and more than $180 \mathrm{mmHg}$, haemoglobin $<8 \mathrm{mg} / \mathrm{dL}$ and neuromusculoskeletal disease of the lower limbs that interferes with the exercise.

The treatment group received a mild intensity intradialytic aerobic exercise program (30-39\% HRR or 11-12 Borg scale) with a cycle ergometer (Terra Fitness $®$ brand) for 30 minutes, starting with a 5-minute warm-up, a 20-minute core phase and a cool-down phase. 5 minutes, exercise frequency 2 times a week according to hemodialysis schedule and carried out for 12 weeks. The control group was not given an aerobic exercise program. Both groups were given education to continue drug therapy and diet as before. Parameters evaluated in this study were serum CRP levels, which were evaluated four times, just pre post HD before the intervention and briefly pre post $\mathrm{HD}$ at the end of the study after 12 weeks of the intervention of the aerobic exercise program. CRP examination using a dimension EXL analyzer (Siemens) at the GDC Clinical Pathology Laboratory of Dr. Soetomo Hospital. All data were analyzed using IBM SPSS version 26. 


\section{Result}

A total of 30 routine haemodialysis patients met the inclusion criteria and did not include the exclusion criteria. Subjects were divided into 2 groups, consisting of 15 subjects in each group. Of the 30 patients, there were 4 patients who dropped out due to illness requiring treatment ( 1 treatment and 1 control) and withdrew from the study for personal reasons (1 treatment and 1 control), so that a total of 26 patients were able to continue until the end of the study.

Table 1 Characteristic subject and comparisons between groups before intervention

Characteristic
Total $n=26$
Group

\section{Intervention (I)} $(\mathrm{n}=13)$

$7(53,84 \%)$

$39.62 \pm 6,31$

$23,36 \pm 4,44$

$15(57,69 \%)$

$38,77 \pm 5,70$

$22,73 \pm 3,81$

BMI (kg/m2)

Systolic BP

Heart rate rest

$157,52 \pm 19,60$

$154,08 \pm 19,62$

$88,56 \pm 13,92$

$91 \pm 14,70$

$0,33 \pm 0,33$

$0,35 \pm 0,32$

CRP pre-HD

CRP post-HD

\section{Control (C)} $(n=13)$

Independent $t$ test, significant difference if $\mathrm{p}<0,05$

* Mann Whitney U test, significant difference if $\mathrm{p}<0,05$

Table 2 Comparison of CRP in Intervention (I) and Control (C) Groups before and after intervention

\begin{tabular}{ccccc}
\hline Variabel & Pre & Post & z-score & p-value \\
\cline { 2 - 3 } & $\mathrm{mg} / \mathrm{dL}$ & $\mathrm{mg} / \mathrm{dL}$ & & \\
CRP IG & $0,33 \pm 0,33$ & $0,27 \pm 0,16$ & $-0,536$ & 0,543 \\
CRP CG & $0,97 \pm 0,83$ & $0,64 \pm 0,91$ & $-1,427$ & 0,080 \\
\hline
\end{tabular}

Mann Whitney U test, significant difference if $\mathrm{p}<0,05$

Males were found more than females in both groups. There were 7 and 8 men in the treatment and control groups, respectively $(53.4 \%$ and $61.53 \%)$. The mean age of the subjects was $39.62 \pm 6.31$ years in the treatment group and $37.92 \pm 5.14$ years in the control group. The independent $t$ test showed that there was no significant difference in the distribution of age data between groups I and C $(p=0.460)$. BMI in the treatment group was $23.36 \pm 4.44 \mathrm{~kg} / \mathrm{m} 2$, while the control group was $22.1 \pm 3.12 \mathrm{~kg} / \mathrm{m} 2$. Independent t test showed that there was no significant difference in the distribution of BMI between the treatment and control groups $(\mathrm{p}=0.579)$.

The Mann Whitney U test analysis showed that there was a significant difference in CRP before the pre HD intervention between the treatment and control groups with a significance value of 0.026. The CRP before the pre HD intervention between the two groups did not show a significant difference with a significance value of 0.064 . 
Comparison of CRP before intervention and after intervention comparing CRP pre-HD (table 2). There was a decrease in the mean CRP after the intervention, from $3.3 \pm 3.3$ to $2.7 \pm 1.6 \mathrm{mg} / \mathrm{L}$, but the pair $\mathrm{t}$ test showed no significant difference between pre and post intervention CRP in the treatment group with a significance value of 0.543 as well as CRP in the control group with a significance value of 0.080 .

\section{Discussion}

There was a decrease in the mean post-intervention CRP compared to the pre-intervention in the treatment group, from $0.33 \pm 0.33 \mathrm{mg} / \mathrm{dL}$ to $0.27 \pm 0.16 \mathrm{mg} / \mathrm{dL}$, but this decrease was not significant $(\mathrm{p}=$ 0.543). This could be due to uncontrollable confounding variables such as BMI, fat mass, diet, albumin levels, stress, comorbidities that could affect CRP levels in this study. Exercise intensity that has not been maximized is prescribed because it is difficult to achieve the intensity according to the target heart rate in the first two months of training (the Borg scale is first achieved in the first two months of training). In addition, low intensity may not be enough to increase fat metabolism which greatly affects CRP . levels.

This population are at greater risk of experiencing inflammatory reactions caused by various factors, including: uremia, dialysis-related and infectious complications. Uraemia will result in decreased cytokine clearance, oxidative stress and accumulation of advanced glycation end products (AGE's). Haemodialysis can increase the inflammatory response due to the presence of bioincompatible dialysis membranes, vascular access infections and endotoxin exposure. Impaired immune response is common in ESRD patients, characterized by hypo responsiveness of neutrophils and T-cells that will increase the risk of infection. These factors will stimulate the inflammatory response by activating the production of Interleukin-1 (IL-1), IL-6, Tumor necrotizing factor- $\alpha$ (TNF- $\alpha$ ) and interferon- $\gamma$ (IFN- $\gamma$ ) by macrophages which will increase C concentration. -Reactive Protein (CRP) serum (Mukai et al., 2018).

C-Reactive Protein is an inflammatory marker that is widely used because it is more affordable and available in most hospitals in developing countries (Abdellah et al., 2020). Immunoassays and laser nephelometry are the methods to quantify CRP levels and are cheap, accurate, and fast. To detect lower levels of CRP (0.3 to $1.0 \mathrm{mg} / \mathrm{L}$ ), high-sensitivity CRP methods are recommended as the usual CRP detection tests are less precise. High-sensitivity CRP only denotes the assay process used, allowing for detection of lower levels of CRP, and not a different, or more specific, differential diagnosis (Nehring et al., 2021). This marker can be used to predict future cardiovascular events. Elevated CRP and other acute phase proteins are one of the biggest risk factors for death in patients with chronic kidney failure (Heidari, 2012). Serum CRP levels of ESRD patients with routine haemodialysis were found to be higher than the age population without ESRD (Abdel-Messeih et al., 2020).

This study showed that $\mathrm{C}$-reactive protein (CRP), as a marker of chronic inflammation in dialysis patients, did not change significantly after 12 weeks of intradialytic exercise. The choice of CRP as a marker of inflammation in our study was due to its strong correlation with cardiovascular prognostic factors. In addition, CRP has a long half-life and is independent of other factors. However, our study showed that subjects' CRP levels had a wide range of variation, which could increase analysis bias between groups. Suhardjono et al., (2019) reported improvements in physical capacity, but there was no significant change in hsCRP levels in ESRD patients on routine haemodialysis after 12 weeks of physical exercise.

Mild CRP elevations can be seen without systemic disease or inflammation. Women and elderly patients have higher CRP levels. Obesity, insomnia, depression, smoking, chronic disease and diabetes can all contribute to mild increases in CRP. Body fat percentage is closely related to CRP. Given the highly variable 
causality of elevated CRP, mild increases in CRP can be difficult to interpret and cannot be used as isolated test results. CRP levels are useful in differentiating infection from inflammation if they are very high, but levels between $1 \mathrm{mg} / \mathrm{dL}$ and $10 \mathrm{mg} / \mathrm{dL}$ can be difficult to interpret accurately. In some studies CRP has been used as a prognostic factor in acute and chronic infections, but a mild increase in CRP may not be clinically significant. Clinical correlation is highly recommended when interpreting CRP test results (Nehring et al., 2021).

\section{Conclusion}

Low-intensity intradialytic aerobic exercise has no effect on serum levels of C-reactive protein in end-stage renal disease patients. Further studies with a larger number of subjects are needed to evaluate the long-term effects of intradialytic aerobic exercise and determine the appropriate exercise intensity for this population. The use of other inflammatory markers such as TNF $\alpha$ and IL6 to assess inflammation in ESRD patients can be considered.

\section{References}

Abdellah AS., Obaya HE., Abdeen dan El Latief .2020. Effect of Intradialytic Aerobic Exercise on C-Reactive Protein for Chronic Kidney Disease. N Y Sci J 2020;13(5):36-42]. ISSN 1554-0200 (print); ISSN 2375-723X. doi:10.7537/marsnys130520.05.

Abdel-Messeih, P. L., Alkady, M. M., Nosseir, N. M., \& Tawfik, M. S. (2020). Inflammatory markers in endstage renal disease patients on haemodialysis. Journal of medical biochemistry, 39(4), 481-487. https://doi.org/10.5937/jomb0-25120

Bikbov B., Purcell C.A., Levey A.S., Smith M., Abdoli A., Abebe M., Adebayo O.M. Murray C.J.L. (2020). Global, regional, and national burden of chronic kidney disease, 1990-2017: a systematic analysis for the Global Burden of Disease Study 2017. The Lancet, 395 (10225) , pp. 709-733

Dungey, M. et al. (2013) 'Inflammatory factors and exercise in Chronic Kidney Disease', International Journal of Endocrinology. doi: 10.1155/2013/569831.

Groussard, C. et al. (2015) 'Beneficial effects of an intradialytic cycling training program in patients with end-stage kidney disease', Applied Physiology, Nutrition and Metabolism. doi: 10.1139/apnm-20140357.

Heidari, B. (2012) 'C-reactive protein and other markers of inflammation in hemodialysis patients', Caspian Journal of Internal Medicine.

Heiwe, S. and Jacobson, S. H. (2014) 'Exercise training in adults with PGK: A systematic review and metaanalysis', American Journal of Kidney Diseases. doi: 10.1053/j.ajkd.2014.03.020.

Johansen, K. L. (2007) 'Exercise in the end-stage renal disease population', Journal of the American Society of Nephrology. doi: 10.1681/ASN.2007010009.

Levin, A. et al. (2013) 'Kidney disease: Improving global outcomes (KDIGO) PGK work group. KDIGO 2012 clinical practice guideline for the evaluation and management of chronic kidney disease', Kidney 
International Supplements. doi: 10.1038/kisup.2012.73.

Lwanga, S.K and Lemeshow, S. 1991. Sample sizes determination in health studies: a practical manual. WHO Library Cataloguing in Publication Data

Mukai, H., Villafuerte, H., Qureshi, A. R., Lindholm, B., \& Stenvinkel, P. (2018). Serum albumin, inflammation, and nutrition in end-stage renal disease: C-reactive protein is needed for optimal assessment. Seminars in dialysis, 31(5), 435-439. https://doi.org/10.1111/sdi.12731

Nehring SM, Goyal A, Bansal P, et al,.2021. C Reactive Protein In: Stat Pearls [Internet]. Treasure Island (FL): StatPearls Publishing

PERNEFRI. (2018). 11 ${ }^{\text {th }}$ Indonesian Renal Registry

Sheng, K. et al. (2014) 'Intradialytic exercise in hemodialysis patients: A systematic review and metaanalysis', American Journal of Nephrology. doi: 10.1159/000368722.

Suhardjono et al. (2019) 'The effect of intradialytic exercise twice a week on the physical capacity, inflammation, and nutritional status of dialysis patients: A randomized controlled trial', Hemodialysis International. doi: 10.1111/hdi.12764. 